\title{
Stabilization of Networked Control Systems with Induced Delays and Actuator Saturation
}

\author{
Luo Zhang, Mou Chen, Qingxian Wu, and Bei Wu \\ College of Automation Engineering, Nanjing University of Aeronautics and Astronautics, Nanjing 210016, China \\ Correspondence should be addressed to Mou Chen; chenmou@nuaa.edu.cn
}

Received 4 February 2016; Accepted 18 July 2016

Academic Editor: Sabri Arik

Copyright (C) 2016 Luo Zhang et al. This is an open access article distributed under the Creative Commons Attribution License, which permits unrestricted use, distribution, and reproduction in any medium, provided the original work is properly cited.

The problem of state feedback stabilization is studied for networked control systems (NCSs) subject to actuator saturation and network-induced delays. To facilitate the controller design, the NCSs are modeled as a class of discrete-time systems with bounded delays and input saturation. Based on Lyapunov-Krasovskii theory and free weighting matrix approach, the sufficient condition is derived in terms of linear matrix inequality for the asymptotic stability. Finally, the effectiveness of the developed control approach is proved through numerical examples.

\section{Introduction}

Control systems whose feedback paths are implemented by communication networks are called networked control systems (NCSs) $[1,2]$. NCSs are widely used in many areas, such as industrial automation, intelligent vehicle systems, flight control, and mobile robotics [3]. In recent years, NCSs have received increasing attention due to the accelerated development of communication techniques, network techniques, and control methods. Network-induced delays and actuator saturation are often encountered in many practical systems including NCSs $[4,5]$. If the time delays and the saturation problem are ignored in system analysis, the control performance of NCSs may deteriorate. Since the existence of time delays and actuator saturation often leads to the instability of NCSs, research on time delay systems with actuator saturation is a topic of great practical and theoretical importance. Time delays are often encountered in various practical engineering systems, such as chemical systems, power systems, and networked control systems [6-8]. In [9], a brief overview of time delay control was provided. The state estimation problem was considered for discrete-time linear systems with Markovian time delay in [10]. In [11], a linear controller was designed to realize chaos control and synchronization for Lorenz system with time-varying lags. Since the introduction of networks, almost all networked control systems have network-induced delays which can make systems unstable. In the last decades, many researchers have studied stability analysis and controller design for stabilization of NCSs in the presence of network-induced delays. In [12], an approach was established to codesign an output feedback controller and a channel-access managing policy for networked control systems. In [13], stochastic optimal controllers were designed for networked control systems whose induced delay was longer than a sampling period. The stabilization problem was studied for a class of networked control systems in the discrete-time domain with random delays in [14]. The robust control problem was studied for a class of stochastic uncertain discrete-time delay systems with missing measurements in [15]. In [16], extensions to continuous NCSs were studied and a design scheme was proposed for the observer-based output feedback controller. In [17], the problem of integrated design of controller and communication sequences was addressed for networked control systems with simultaneous consideration of medium access limitations and network-induced delays, packet dropouts, and measurement quantization. Although NCSs with time delays were widely investigated, actuator saturation problems in NCSs were seldom considered in the existing research results which needs to be further studied.

In practical systems, due to some physical limitations, the saturation problem is inevitable. In recent years, saturated systems have become a popular research topic. In [18], adaptive output feedback control was studied for a kind of 


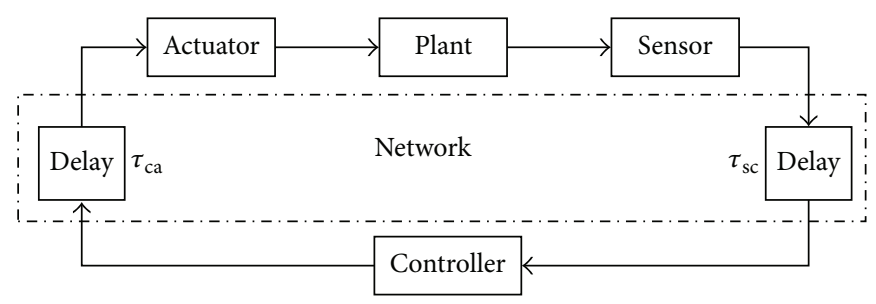

FIGURE 1: NCS model.

uncertain system in the presence of saturation. The problem was studied for stabilizing a linear system with delayed and saturating feedback in [19]. In [20, 21], LMI-based methods were presented for regional stability and performance of linear antiwindup compensators for linear control systems. In [22], the design of guaranteed transient performance based attitude control was described for the near space vehicle (NSV) with control input saturation using backstepping method. A method was proposed for the analysis and control design of linear systems in the presence of actuator saturation and disturbances in [23]. In [24], robust control was studied for uncertain linear system subject to input saturation. Adaptive tracking control problem was investigated for uncertain nonlinear systems with input saturation and unknown external disturbances in [25]. In [26], an actuator robust fault tolerant control was proposed for ocean surface vessels with parametric uncertainties, unknown disturbances, and input saturation. During the last few years, problems with actuator saturation have been extended to singular systems [27], Markovian jump systems [28], decentralized control systems [29], and Hamilton systems [30]. The problem of adaptive control of linear discrete-time systems with actuator saturation and unknown parameters was investigated in [31]. In [32], a novel adaptive neural network control approach was presented for a class of uncertain discrete-time nonlinear strict-feedback systems with input saturation. However, time delays and saturation problems were seldom considered in NCSs simultaneously.

This work is motivated by the stabilization control of networked control systems with network-induced delays and actuator saturation. Based on Lyapunov-Krasovskii stability theory and free weighting matrix approach, stability analysis and a state feedback controller of NCSs are studied.

The organization of the paper is as follows. Section 2 describes networked control systems (NCSs) with networkinduced delays and actuator saturation and details the problem formulation. Section 3 gives stability analysis and stabilization approach of NCSs. Numerical simulations are presented in Section 4 to demonstrate the effectiveness of the developed control method. The conclusion is given in Section 5.

\section{Problem Formulation}

In this section, system modeling of a kind of NCS with actuator saturation is considered. As illustrated in Figure 1 [3], NCS consists of four components: a plant, an actuator, a controller, and a sensor. A certain communication network connects these components.
In practical NCSs, state and input delays of control plant usually exist. Thus, the control plant of NCSs can be modeled as a class of discrete systems with state, input delays, and input saturation which can be described by

$$
\begin{aligned}
x(k+1)= & A x(k)+A_{d} x(k-d(k))+B \text { sat }(u(k)) \\
& +B_{h} \text { sat }(u(k-h(k))),
\end{aligned}
$$

where $x(k) \in \mathfrak{R}^{n}$ is the state vector of the system and $u(k) \epsilon$ $\mathfrak{R}^{m}$ is the control input signal. $d(k)$ is state delay with lower and upper bounds $0 \leq d_{1} \leq d(k) \leq d_{2}$ and $h(k)$ is input delay which satisfies $0 \leq h_{1} \leq h(k) \leq h_{2}$. $A, A_{d}, B$, and $B_{h}$ are known real matrices with appropriate dimensions. The control input sat $(u(k))$ is required to meet the saturation levels where sat $(\cdot)$ is a saturation function vector defined as

$$
\text { sat }(u)=\left[\operatorname{sat}\left(u_{1}\right) \text {, sat }\left(u_{2}\right), \ldots, \text { sat }\left(u_{m}\right)\right]^{T} \text {, }
$$

with $\operatorname{sat}\left(u_{j}\right)=\operatorname{sign}\left(u_{j}\right) \max \left\{u_{j \max },\left|u_{j}\right|\right\}, j \in[1, m]$, and a saturation boundary $u_{j \max }$.

In the NCSs, the time delay is composed of two parts: $\tau^{\mathrm{ca}}$ is generated from the controller to the actuator and $\tau^{\mathrm{sc}}$ is produced from the sensor to the controller. Compared with these two parts of time delays, the calculation time of controller, actuator, and sensor is small enough so that it often can be ignored. To design the controller, the following assumptions are needed.

Assumption 1 (see [33]). The sensor is time driven. The controller and actuator are event driven.

Assumption 2 (see [33]). The data transmission is with a single packet and there are no packet dropouts.

In this paper, a state feedback controller is designed as $u(k)=2 K x\left(k-\tau_{\mathrm{ca}}-\tau_{\mathrm{sc}}\right)$. We combine $\tau_{\mathrm{ca}}$ and $\tau_{\mathrm{sc}}$ to $\tau(k)$. Then, the state feedback controller is rewritten as follows:

$$
u(k)=2 K x(k-\tau(k)),
$$

where $K$ is the state feedback controller gain and $\tau(k)$ is network-induced delay which is time varying and satisfies $0 \leq \tau_{1} \leq \tau(k) \leq \tau_{2}$.

Under controller (3), define $\eta_{1}(k)=$ sat $(2 K x(k-\tau(k)))-$ $K x(k-\tau(k))$ and $\eta_{2}(k)=$ sat $(2 K x(k-\tau(k)-h(k)))-K x(k-$ $\tau(k)-h(k))$. Then, the closed-loop system can be written as follows:

$$
\begin{aligned}
x(k+1)= & A x(k)+A_{d} x(k-d(k)) \\
& +B K x(k-\tau(k)) \\
& +B_{h} K x(k-\tau(k)-h(k))+B \eta_{1}(k) \\
& +B_{h} \eta_{2}(k) .
\end{aligned}
$$


Before presenting the main results of this paper, we need to introduce the following lemmas which are crucial to our main results.

Lemma 3 (see [34]). Given symmetric constant matrices $S_{11}$, $S_{22}$ and constant matrix $S_{12}$ of approximate dimensions, then $S_{22}<0$ and $S_{11}-S_{12} S_{22}^{-1} S_{12}^{T}<0$ holds if and only if

$$
\left[\begin{array}{ll}
S_{11} & S_{12} \\
S_{12}^{T} & S_{22}
\end{array}\right]<0 .
$$

Lemma 4 (see [35]). Considering $\eta_{1}(k)$ and $\eta_{2}(k)$ which are defined before, one has the following inequalities:

$$
\begin{aligned}
& \eta_{1}^{T}(k) \eta_{1}(k) \leq x^{T}(k-\tau(k)) K^{T} K x(k-\tau(k)), \\
& \eta_{2}^{T}(k) \eta_{2}(k) \\
& \quad \leq x^{T}(k-\tau(k)-h(k)) K^{T} K x(k-\tau(k)-h(k)) .
\end{aligned}
$$

Proof. Let $\eta(k)=\left[\eta_{1}^{T}(k), \eta_{2}^{T}(k), \ldots, \eta_{m}^{T}(k)\right]^{T}$ and $K=$ $\left[K_{1}^{T}, K_{2}^{T}, \ldots, K_{m}^{T}\right]^{T}$. According to (2) and (3), the following is yielded:

$$
\begin{aligned}
& \operatorname{sat}\left(2 K_{j} x(k-\tau(k))\right)=\operatorname{sat}\left(u_{j}(k-\tau(k))\right) \\
&= \operatorname{sign}\left(2 K_{j} x(k-\tau(k))\right) \\
& \cdot \max \left(u_{j \max },\left|2 K_{j} x(k-\tau(k))\right|\right) .
\end{aligned}
$$

(1) If $2 K_{j} x(k-\tau(k)) \geq u_{j \max }>0$, we have

$$
\begin{aligned}
& \text { sat }\left(2 K_{j} x(k-\tau(k))\right)-K_{j} x(k-\tau(k)) \\
& \leq 2 K_{j} x(k-\tau(k))-K_{j} x(k-\tau(k)) \\
& \quad=K_{j} x(k-\tau(k)) \Longrightarrow \\
& \left|\eta_{1 j}(k)\right|=\left|\operatorname{sat}\left(2 K_{j} x(k-\tau(k))\right)-K_{j} x(k-\tau(k))\right| \\
& \quad \leq\left|K_{j} x(k-\tau(k))\right| \Longrightarrow \\
& \eta_{1 j}^{2}(k)=\left(\operatorname{sat}\left(2 K_{j} x(k-\tau(k))\right)-K_{j} x(k-\tau(k))\right)^{2} \\
& \leq\left(K_{j} x(k-\tau(k))\right)^{2} .
\end{aligned}
$$

Hence, one has

$$
\begin{aligned}
& \begin{array}{r}
\eta_{1}^{T}(k) \eta_{1}(k)=\sum_{j=1}^{m} \eta_{1 j}^{2}(k) \leq \sum_{j=1}^{m}\left(K_{j} x(k-\tau(k))\right)^{2} \\
=x^{T}(k-\tau(k)) K^{T} K x(k-\tau(k))
\end{array} \\
& \text { (2) If }-u_{j \max } \leq 2 K_{j} x(k-\tau(k)) \leq u_{j \max }, \text { we have } \\
& \eta_{1 j}(k)=\operatorname{sat}\left(2 K_{j} x(k-\tau(k))\right)-K_{j} x(k-\tau(k)) \\
& =K_{j} x(k-\tau(k)) \Longrightarrow \\
& \eta_{1 j}^{2}(k)=\left(\operatorname{sat}\left(2 K_{j} x(k-\tau(k))\right)-K_{j} x(k-\tau(k))\right)^{2} \\
& =\left(K_{j} x(k-\tau(k))\right)^{2}
\end{aligned}
$$

Hence, one has

$$
\begin{aligned}
& \eta_{1}^{T}(k) \eta_{1}(k)=x^{T}(k-\tau(k)) K^{T} K x(k-\tau(k)) . \\
& \text { (3) If } 2 K_{j} x(k-\tau(k)) \leq-u_{j \max }<0 \text {, this yields } \\
& \text { sat }\left(2 K_{j} x(k-\tau(k))\right)-K_{j} x(k-\tau(k)) \\
& \quad \geq 2 K_{j} x(k-\tau(k))-K_{j} x(k-\tau(k)) \\
& \quad=K_{j} x(k-\tau(k)), \\
& \text { sat }\left(2 K_{j} x(k-\tau(k))\right)-K_{j} x(k-\tau(k)) \\
& \quad<-K_{j} x(k-\tau(k)) \Longrightarrow \\
& \quad \eta_{j}(k)|=| \operatorname{sat}\left(2 K_{j} x(k-\tau(k))\right)-K_{j} x(k-\tau(k)) \mid \\
& \quad \leq\left|-K_{j} x(k-\tau(k))\right| \Longrightarrow \\
& \quad \leq\left(K_{j} x(k-\tau(k))\right)^{2} . \\
& \left(\eta_{j}(k)\right)^{2} \\
& \quad\left(\operatorname{sat}\left(2 K_{j} x(k-\tau(k))\right)-K_{j} x(k-\tau(k))\right)^{2} \\
& \quad
\end{aligned}
$$

Hence, we have

$$
\begin{aligned}
\eta_{1}^{T}(k) \eta_{1}(k) & =\sum_{j=1}^{m} \eta_{j}^{2}(k) \leq \sum_{j=1}^{m}\left(K_{j} x(k-\tau(k))\right)^{2} \\
& =x^{T}(k-\tau(k)) K^{T} K x(k-\tau(k)) .
\end{aligned}
$$

Combining (9), (11), and (13), we have $\eta_{1}^{T}(k) \eta_{1}(k) \leq x^{T}(k-$ $\tau(k)) K^{T} K x(k-\tau(k))$. Similarly, we can prove $\eta_{2}^{T}(k) \eta_{2}(k) \leq$ $x^{T}(k-\tau(k)-h(k)) K^{T} K x(k-\tau(k)-h(k))$. This completes the proof.

Our main purpose of this paper is to design a state feedback controller for the networked control system (1) such that the closed-loop system (4) is asymptotically stable with bounded network-induced delays and actuator saturation.

\section{Main Results}

In this section, we obtained a sufficient condition for the asymptotical stability of system (1) and the synthesis of controller design will be established. The asymptotical stability criteria are given in Theorem 5 .

Theorem 5. The closed-loop NCS is asymptotically stable under controller (3) if there exist symmetric matrices $P>$ $0, Q_{i}>0, S_{i}>0, i=1,2,3, X>0, \bar{X}>0, \widetilde{X}>0$ and 
matrices $N, M, T$ with appropriate dimensions such that the following matrix inequalities hold:

$$
\begin{aligned}
& \Xi=\left[\begin{array}{cccccc}
\Xi_{11} & \Xi_{12} & \Xi_{13} & \Xi_{14} & \Xi_{15} & \Xi_{16} \\
* & \Xi_{22} & \Xi_{23} & \Xi_{24} & \Xi_{25} & \Xi_{26} \\
* & * & \Xi_{33} & \Xi_{34} & \Xi_{35} & \Xi_{36} \\
* & * & * & \Xi_{44} & \Xi_{45} & \Xi_{46} \\
* & * & * & * & \Xi_{55} & \Xi_{56} \\
* & * & * & * & * & \Xi_{66}
\end{array}\right]<0, \\
& \psi_{1}=\left[\begin{array}{cc}
X & N \\
* & Q_{1}
\end{array}\right]>0, \\
& \psi_{2}=\left[\begin{array}{cc}
\bar{X} & M \\
* & Q_{2}
\end{array}\right]>0, \\
& \psi_{3}=\left[\begin{array}{cc}
\widetilde{X} & T \\
* & Q_{3}
\end{array}\right]>0,
\end{aligned}
$$

where * is used as an ellipse for terms induced by symmetry and

$$
\begin{aligned}
\Xi_{11}= & +P(A-I)^{T}+(A-I)^{T} P \\
& +(A-I)^{T} H(A-I)+N_{1}^{T}+N_{1}+M_{1}^{T} \\
& +M_{1}+T_{1}^{T}+T_{1}+d_{2} X_{11}+\tau_{2} \bar{X}_{11} \\
& +\left(\tau_{2}+h_{2}\right) \widetilde{X}_{11}, \\
\Xi_{12}= & P A_{d}+(A-I)^{T} H A_{d}-N_{1}+N_{2}^{T}+d_{2} X_{12} \\
& +\tau_{2} \bar{X}_{12}+\left(\tau_{2}+h_{2}\right) \widetilde{X}_{12}, \\
\Xi_{13}= & P B K+(A-I)^{T} H B K-M_{1}+M_{2}^{T}+d_{2} X_{13} \\
& +\tau_{2} \bar{X}_{13}+\left(\tau_{2}+h_{2}\right) \widetilde{X}_{13}, \\
\Xi_{14}= & P B_{d} K+(A-I)^{T} H B_{d} K-T_{1}+T_{2}^{T}+d_{2} X_{14} \\
& +\tau_{2} \bar{X}_{14}+\left(\tau_{2}+h_{2}\right) \widetilde{X}_{14}, \\
& +\left(\tau_{2}+h_{2}\right) \widetilde{X}_{22}, \\
\Xi_{15}= & P B+(A-I)^{T} H B+d_{2} X_{15}+\tau_{2} \bar{X}_{15} \\
& +\left(\tau_{2}+h_{2}\right) \widetilde{X}_{15}, \\
\Xi_{25}= & A_{d}^{T} H B+d_{2} X_{25}+\tau_{2} \bar{X}_{25}+\left(\tau_{2}+h_{2}\right) \widetilde{X}_{25}, \\
\Xi_{22}= & A_{d}^{T} H A A_{d}^{T} H B K+d_{2} X_{23}+\tau_{2} \bar{X}_{23}+\left(\tau_{2}+h_{2}\right) \widetilde{X}_{23}, \\
\Xi_{16}= & P B_{d}+(A-I)^{T} H B_{d}+d_{2} X_{16}+\tau_{2} \bar{X}_{16} \\
& +\tau_{2}+d_{2} X_{22}+\tau_{2} \bar{X}_{22}, \\
& +d_{2} X_{24}+\tau_{2} \bar{X}_{24}+\left(\tau_{2}+h_{2}\right) \widetilde{X}_{24}, \\
& +(A)
\end{aligned}
$$

$$
\begin{aligned}
& \Xi_{26}=A_{d}^{T} H B_{d}+d_{2} X_{26}+\tau_{2} \bar{X}_{26}+\left(\tau_{2}+h_{2}\right) \widetilde{X}_{26}, \\
& \Xi_{33}=(B K)^{T} H(B K)-S_{2}-M_{2}^{T}-M_{2}+\alpha_{1} K^{T} K \\
& +d_{2} X_{33}+\tau_{2} \bar{X}_{33}+\left(\tau_{2}+h_{2}\right) \widetilde{X}_{33} \text {, } \\
& \Xi_{34}=(B K)^{T} H\left(B_{d} K\right)+d_{2} X_{34}+\tau_{2} \bar{X}_{34} \\
& +\left(\tau_{2}+h_{2}\right) \widetilde{X}_{34} \\
& \Xi_{35}=(B K)^{T} H B+d_{2} X_{35}+\tau_{2} \bar{X}_{35}+\left(\tau_{2}+h_{2}\right) \widetilde{X}_{35}, \\
& \Xi_{36}=(B K)^{T} H B_{d}+d_{2} X_{36}+\tau_{2} \bar{X}_{36}+\left(\tau_{2}+h_{2}\right) \widetilde{X}_{36} \text {, } \\
& \Xi_{44}=\left(B_{d} K\right)^{T} H B_{d} K-S_{3}-T_{2}-T_{2}^{T}+\alpha_{2} K^{T} K \\
& +d_{2} X_{44}+\tau_{2} \bar{X}_{44}+\left(\tau_{2}+h_{2}\right) \widetilde{X}_{44}, \\
& \Xi_{45}=\left(B_{d} K\right)^{T} H B+d_{2} X_{45}+\tau_{2} \bar{X}_{45}+\left(\tau_{2}+h_{2}\right) \widetilde{X}_{45} \text {, } \\
& \Xi_{46}=\left(B_{d} K\right)^{T} H B_{d}+d_{2} X_{46}+\tau_{2} \bar{X}_{46}+\left(\tau_{2}+h_{2}\right) \widetilde{X}_{46} \text {, } \\
& \Xi_{55}=B^{T} H B-\alpha_{1} I+d_{2} X_{55}+\tau_{2} \bar{X}_{55}+\left(\tau_{2}+h_{2}\right) \widetilde{X}_{55} \text {, } \\
& \Xi_{56}=B^{T} H B_{d}+d_{2} X_{56}+\tau_{2} \bar{X}_{56}+\left(\tau_{2}+h_{2}\right) \widetilde{X}_{56} \text {, } \\
& \Xi_{66}=B_{d}^{T} H B_{d}-\alpha_{2} I+d_{2} X_{66}+\tau_{2} \bar{X}_{66} \\
& +\left(\tau_{2}+h_{2}\right) \widetilde{X}_{66} \text {, } \\
& H=P+d_{2} Q_{1}+\tau_{2} Q_{2}+\left(\tau_{2}+h_{2}\right) Q_{3} \text {, } \\
& J=\left(d_{2}-d_{1}+1\right) S_{1}+\left(\tau_{2}-\tau_{1}+1\right) S_{2} \\
& +\left(\left(\tau_{2}+h_{2}\right)-\left(\tau_{1}+h_{1}\right)+1\right) S_{3}, \\
& X=\left[\begin{array}{cccccc}
X_{11} & X_{12} & X_{13} & X_{14} & X_{15} & X_{16} \\
* & X_{22} & X_{23} & X_{24} & X_{25} & X_{26} \\
* & * & X_{33} & X_{34} & X_{35} & X_{36} \\
* & * & * & X_{44} & X_{45} & X_{46} \\
* & * & * & * & X_{55} & X_{56} \\
* & * & * & * & * & X_{66}
\end{array}\right] \text {, } \\
& \bar{X}=\left[\begin{array}{cccccc}
\bar{X}_{11} & \bar{X}_{12} & \bar{X}_{13} & \bar{X}_{14} & \bar{X}_{15} & \bar{X}_{16} \\
* & \bar{X}_{22} & \bar{X}_{23} & \bar{X}_{24} & \bar{X}_{25} & \bar{X}_{26} \\
* & * & \bar{X}_{33} & \bar{X}_{34} & \bar{X}_{35} & \bar{X}_{36} \\
* & * & * & \bar{X}_{44} & \bar{X}_{45} & \bar{X}_{46} \\
* & * & * & * & \bar{X}_{55} & \bar{X}_{56} \\
* & * & * & * & * & \bar{X}_{66}
\end{array}\right], \\
& \widetilde{X}=\left[\begin{array}{cccccc}
\widetilde{X}_{11} & \widetilde{X}_{12} & \widetilde{X}_{13} & \widetilde{X}_{14} & \widetilde{X}_{15} & \widetilde{X}_{16} \\
* & \widetilde{X}_{22} & \widetilde{X}_{23} & \widetilde{X}_{24} & \widetilde{X}_{25} & \widetilde{X}_{26} \\
* & * & \widetilde{X}_{33} & \widetilde{X}_{34} & \widetilde{X}_{35} & \widetilde{X}_{36} \\
* & * & * & \widetilde{X}_{44} & \widetilde{X}_{45} & \widetilde{X}_{46} \\
* & * & * & * & \widetilde{X}_{55} & \widetilde{X}_{56} \\
* & * & * & * & * & \widetilde{X}_{66}
\end{array}\right] \text {, }
\end{aligned}
$$




$$
\begin{aligned}
N & =\left[\begin{array}{llllll}
N_{1}^{T} & N_{2}^{T} & 0 & 0 & 0 & 0
\end{array}\right]^{T}, \\
M & =\left[\begin{array}{llllll}
M_{1}^{T} & 0 & M_{2}^{T} & 0 & 0 & 0
\end{array}\right]^{T}, \\
T & =\left[\begin{array}{llllll}
T_{1}^{T} & 0 & 0 & T_{2}^{T} & 0 & 0
\end{array}\right]^{T} .
\end{aligned}
$$

Proof. In order to analyze the closed-loop system stability, the Lyapunov-Krasovskii functional candidate is constructed as follows:

$$
\begin{aligned}
V(k)= & V_{1}(k)+V_{2}(k)+V_{3}(k)+V_{4}(k)+V_{5}(k) \\
& +V_{6}(k)+V_{7}(k),
\end{aligned}
$$

where

$$
\begin{aligned}
& V_{1}(k)=x^{T}(k) P x(k), \\
& V_{2}(k)=\sum_{\theta=-d_{2}+1}^{0} \sum_{l=k-1+\theta}^{k-1} y^{T}(l) Q_{1} y(l), \\
& V_{3}(k)=\sum_{\theta=-d_{2}+1}^{-d_{1}+1} \sum_{l=k-1+\theta}^{k-1} x^{T}(l) S_{1} x(l), \\
& V_{4}(k)=\sum_{\theta=-\tau_{2}+1} \sum_{l=k-1+\theta}^{k-1} y^{T}(l) Q_{2} y(l), \\
& V_{5}(k)=\sum_{\theta=-\tau_{2}+1} \sum_{l=k-1+\theta}^{k-1} x^{T}(l) S_{2} x(l), \\
& V_{6}(k)=\sum_{\theta=-\tau_{2}-h_{2}+1} \sum_{l=k-1+\theta}^{k-1} y^{T}(l) Q_{3} y(l), \\
& V_{7}(k)=\sum_{\theta=-\tau_{2}-h_{2}+1} \sum_{l=k-1+\theta}^{k-1} x^{T}(l) S_{3} x(l), \\
& y(l)=x(l+1)-x(l) .
\end{aligned}
$$

The time shift of $V(k)$ can be written as follows:

$$
\begin{aligned}
& \Delta V(k)=\sum_{i=1}^{7} \Delta V_{i}(k) \\
& \Delta V_{1}(k)=2 x^{T}(k) P y(k)+y^{T}(k) P y(k), \\
& \Delta V_{2}(k)=d_{2} y^{T}(k) Q_{1} y(k)-\sum_{l=k-d_{2}}^{k-1} y^{T}(l) Q_{1} y(l) \\
& \leq d_{2} y^{T}(k) Q_{1} y(k)-\sum_{l=k-d_{2}(k)}^{k-1} y^{T}(l) Q_{1} y(l),
\end{aligned}
$$

$$
\Delta V_{3}(k)
$$$$
=\left(d_{2}-d_{1}+1\right) x^{T}(k) S_{1} x(k)-\sum_{l=k-d_{2}}^{k-d_{1}} x^{T}(l) S_{1} x(l)
$$

$$
\begin{gathered}
\leq\left(d_{2}-d_{1}+1\right) x^{T}(k) S_{1} x(k) \\
\quad-x^{T}(k-d(k)) S_{1} x(k-d(k)), \\
\Delta V_{4}(k)=\tau_{2} y^{T}(k) Q_{2} y(k)-\sum_{l=k-\tau_{2}}^{k-1} y^{T}(l) Q_{2} y(l) \\
\leq \tau_{2} y^{T}(k) Q_{2} y(k)-\sum_{l=k-\tau(k)}^{k-1} y^{T}(l) Q_{2} y(l),
\end{gathered}
$$

$\Delta V_{5}(k)$

$\leq\left(\tau_{2}-\tau_{1}+1\right) x^{T}(k) S_{2} x(k)$

$$
-x^{T}(k-\tau(k)) S_{2} x(k-\tau(k)),
$$

$\Delta V_{6}(k)$

$$
\begin{aligned}
& \leq\left(\tau_{2}+h_{2}\right) y^{T}(k) Q_{3} y(k) \\
& -\sum_{l=k-\tau(k)-h(k)}^{k-1} y^{T}(l) Q_{3} y(l),
\end{aligned}
$$

$\Delta V_{7}(k)$

$$
\begin{aligned}
\leq & \left(\left(\tau_{2}+h_{2}\right)-\left(\tau_{1}+h_{1}\right)+1\right) x^{T}(k) S_{3} x(k) \\
& -x^{T}(k-\tau(k)-h(k)) S_{3} x(k-\tau(k)-h(k)) .
\end{aligned}
$$

From (27), we can conclude that

$$
\begin{aligned}
\Delta V(k) & \\
\leq & 2 x^{T}(k) P y(k)+y^{T}(k) P y(k)+y^{T}(k) H y(k) \\
& +x^{T}(k) J x(k)-\sum_{l=k-d(k)}^{k-1} y^{T}(k) Q_{1} y(k) \\
& -\sum_{l=k-\tau(k)}^{k-1} y^{T}(l) Q_{2} y(l) \\
& -\sum_{l=k-\tau(k)-h(k)}^{k-1} y^{T}(l) Q_{3} y(l) \\
& -x^{T}(k-d(k)) S_{1} x(k-d(k)) \\
& -x^{T}(k-\tau(k)) S_{2} x(k-\tau(k)) \\
& -x^{T}(k-\tau(k)-h(k)) S_{2} x(k-\tau(k)-h(k)) .
\end{aligned}
$$

For any matrices $N_{i}, M_{i}, T_{i}, i=1,2$, with appropriate dimensions, we have

$$
\begin{aligned}
& 2\left[x^{T}(k) N_{1}+x^{T}(k-d(k)) N_{2}\right] \\
& \quad\left[x(k)-x(k-d(k))-\sum_{l=k-d(k)}^{k-1} y(l)\right]=0,
\end{aligned}
$$




$$
\begin{aligned}
& 2\left[x^{T}(k) M_{1}+x^{T}(k-\tau(k)) M_{2}\right] \\
& \cdot\left[x(k)-x(k-\tau(k))-\sum_{l=k-\tau(k)}^{k-1} y(l)\right]=0, \\
& 2\left[x^{T}(k) T_{1}+x^{T}(k-\tau(k)-h(k)) T_{2}\right] \\
& \cdot\left[x(k)-x(k-\tau(k)-h(k))-\sum_{l=k-\tau(k)-h(k)}^{k-1} y(l)\right] \\
& \quad=0 .
\end{aligned}
$$

Then, we denote matrix $X>0, \bar{X}>0, \widetilde{X}>0$ with appropriate dimensions which are described in Theorem 5 . Let $\zeta_{1}(k)=\left[\begin{array}{llll}x^{T}(k) & x^{T}(k-h(k)) & x^{T}(k-\tau(k)) & x^{T}(k-\tau(k)-\end{array}\right.$ $\left.d(k)) \eta_{1}^{T}(k) \eta_{2}^{T}(k)\right]^{T}$ and $\zeta_{2}(k, l)=\left[\begin{array}{lll}\zeta_{1}^{T}(k) & y^{T}(l)\end{array}\right]^{T}$; we can obtain the following:

$$
\begin{aligned}
& d_{2} \zeta_{1}^{T}(k) X \zeta_{1}(k)-\sum_{l=k-d(k)}^{k-1} \zeta_{1}^{T}(k) X \zeta_{1}(k)>0, \\
& \tau_{2} \zeta_{1}^{T}(k) \bar{X} \zeta_{1}(k)-\sum_{l=k-\tau(k)}^{k-1} \zeta_{1}^{T}(k) \bar{X} \zeta_{1}(k)>0, \\
& \left(\tau_{2}+h_{2}\right) \zeta_{1}^{T}(k) \widetilde{X} \zeta_{1}(k)-\sum_{l=k-\tau(k)-h(k)}^{k-1} \zeta_{1}^{T}(k) \widetilde{X} \zeta_{1}(k) \\
& >0 .
\end{aligned}
$$

From Lemma 4, we have

$$
\begin{aligned}
& \zeta_{1}^{T}(k)\left[\begin{array}{llllll}
0 & 0 & \alpha_{1} K^{T} K & 0 & -\alpha_{1} I & 0
\end{array}\right] \zeta_{1}(k)>0, \\
& \zeta_{1}^{T}(k)\left[\begin{array}{llllll}
0 & 0 & 0 & \alpha_{2} K^{T} K & 0 & -\alpha_{2} I
\end{array}\right] \zeta_{1}(k)>0,
\end{aligned}
$$

where $\alpha_{1}, \alpha_{2}$ are positive scalars.
Considering (28) (29), (30), and (31), we obtain the following:

$$
\begin{aligned}
\Delta V(k)< & \zeta_{1}^{T}(k) \Xi \zeta_{1}(k)-\sum_{l=k-d(k)}^{k-1} \zeta_{2}^{T}(k, l) \psi_{1} \zeta_{2}(k, l) \\
& -\sum_{l=k-\tau(k)}^{k-1} \zeta_{2}^{T}(k, l) \psi_{2} \zeta_{2}(k, l) \\
& -\sum_{l=k-\tau(k)-h(k)}^{k-1} \zeta_{2}^{T}(k, l) \psi_{3} \zeta_{2}(k, l) .
\end{aligned}
$$

It is clear that $\Delta V(k)<0$ is true if the conditions in Theorem 5 are satisfied. The proof is completed.

Theorem 5 gives the sufficient condition for the stability of system (1). However, it should be noted that the inequalities in Theorem 5 are not LMIs and are not easy to deal with. Then, in the following, we present LMI-based criteria to design a state feedback controller for NCSs with induced delays and actuator saturation.

Theorem 6. For given nonzero scalars $\varepsilon_{1}, \varepsilon_{2}, \varepsilon_{3}$ and positive scalars $\alpha_{1}, \alpha_{2}$, if there exist positive symmetric matrices $L, \bar{S}_{i}, X^{\prime}, \bar{X}^{\prime}, \widetilde{X}^{\prime}, i=1,2,3$, and matrices with appropriate dimensions $\bar{N}_{1}, \bar{N}_{2}, \bar{M}_{1}, \bar{M}_{2}, \bar{T}_{1}, \bar{T}_{2}, R$ satisfying the following LMIs:

$$
\begin{aligned}
& \widetilde{\Phi}<0, \\
& \bar{\psi}_{1}=\left[\begin{array}{cc}
X^{\prime} & \bar{N} \\
* & \varepsilon_{1} L
\end{array}\right]>0, \\
& \bar{\psi}_{2}=\left[\begin{array}{cc}
\bar{X}^{\prime} & \bar{M} \\
* & \varepsilon_{2} L
\end{array}\right]>0, \\
& \bar{\psi}_{3}=\left[\begin{array}{cc}
\widetilde{X}^{\prime} & \bar{T} \\
* & \varepsilon_{3} L
\end{array}\right]>0,
\end{aligned}
$$

then a state feedback controller $u(k)=2 K x(k-\tau(k))=$ $2 R L^{-1} x(k-\tau(k))$ can be obtained such that the closed-loop system is asymptotically stable, where

$$
\widetilde{\Phi}=\left[\begin{array}{cccccccccccc}
\widetilde{\Phi}_{11} & \widetilde{\Phi}_{12} & \widetilde{\Phi}_{13} & \widetilde{\Phi}_{14} & \widetilde{\Phi}_{15} & \widetilde{\Phi}_{16} & L(A-I)^{T} & d_{2} L(A-I)^{T} & \tau_{2} L(A-I)^{T} & \left(\tau_{2}+h_{2}\right) L(A-I)^{T} & 0 & 0 \\
* & \widetilde{\Phi}_{22} & \widetilde{\Phi}_{23} & \widetilde{\Phi}_{24} & \widetilde{\Phi}_{25} & \widetilde{\Phi}_{26} & L A_{d}^{T} & d_{2} L A_{d}^{T} & \tau_{2} L A_{d}^{T} & \left(\tau_{2}+h_{2}\right) L A_{d}^{T} & 0 & 0 \\
* & * & \widetilde{\Phi}_{33} & \widetilde{\Phi}_{34} & \widetilde{\Phi}_{35} & \widetilde{\Phi}_{36} & R^{T} B^{T} & d_{2} R^{T} B^{T} & \tau_{2} R^{T} B^{T} & \left(\tau_{2}+h_{2}\right) R^{T} B^{T} & R^{T} & 0 \\
* & * & * & \widetilde{\Phi}_{44} & \widetilde{\Phi}_{45} & \widetilde{\Phi}_{46} & R^{T} B_{h}^{T} & d_{2} R^{T} B_{d}^{T} & \tau_{2} R^{T} B_{d}^{T} & \left(\tau_{2}+h_{2}\right) R^{T} B_{d}^{T} & 0 & R^{T} \\
* & * & * & * & \widetilde{\Phi}_{55} & \widetilde{\Phi}_{56} & L B^{T} & d_{2} L B^{T} & \tau_{2} L B^{T} & \left(\tau_{2}+h_{2}\right) L B^{T} & 0 & 0 \\
* & * & * & * & * & \widetilde{\Phi}_{66} & L B_{h}^{T} & d_{2} L B_{h}^{T} & \tau_{2} L B_{h}^{T} & \left(\tau_{2}+h_{2}\right) L B_{h}^{T} & 0 & 0 \\
* & * & * & * & * & * & -L & 0 & 0 & 0 & 0 & 0 \\
* & * & * & * & * & * & * & -h_{2} \varepsilon_{1}^{-1} L & 0 & 0 & 0 & 0 \\
* & * & * & * & * & * & * & * & -\tau_{2} \varepsilon_{2}^{-1} L & 0 & 0 & 0 \\
* & * & * & * & * & * & * & * & * & -\left(\tau_{2}+h_{2}\right) \varepsilon_{3}^{-1} L & 0 & 0 \\
* & * & * & * & * & * & * & * & * & * & -\alpha_{1}{ }^{-1} I & 0 \\
* & * & * & * & * & * & * & * & * & 0 & -\alpha_{2}{ }^{-1} I
\end{array}\right]
$$


Mathematical Problems in Engineering

7

$$
\begin{aligned}
& \widetilde{\Phi}_{11}=\left(d_{2}-d_{1}+1\right) \bar{S}_{1}+\left(\tau_{2}-\tau_{1}+1\right) \bar{S}_{2}+\left(\left(\tau_{2}+h_{2}\right)-\left(\tau_{1}+h_{1}\right)+1\right) \bar{S}_{3}+(A-I) L+L(A-1)^{T}+\bar{N}_{1}^{T}+\bar{N}_{1}+\bar{M}_{1}^{T} \\
& +\bar{M}_{1}+\bar{T}_{1}^{T}+\bar{T}_{1}+h_{2} X_{11}^{\prime}+\tau_{2} \bar{X}_{11}^{\prime}+\left(\tau_{2}+d_{2}\right) \widetilde{X}_{11}^{\prime} \\
& \widetilde{\Phi}_{12}=A_{d} L-\bar{N}_{1}+\bar{N}_{2}^{T}+d_{2} X_{12}^{\prime}+\tau_{2} \bar{X}_{12}^{\prime}+\left(\tau_{2}+h_{2}\right) \widetilde{X}_{12}^{\prime} \\
& \widetilde{\Phi}_{13}=B R-\bar{M}_{1}+\bar{M}_{2}^{T}+d_{2} X_{13}^{\prime}+\tau_{2} \bar{X}_{13}^{\prime}+\left(\tau_{2}+h_{2}\right) \widetilde{X}_{13}^{\prime} \\
& \widetilde{\Phi}_{14}=B_{h} R-\bar{T}_{1}+\bar{T}_{2}^{T}+d_{2} X_{14}^{\prime}+\tau_{2} \bar{X}_{14}^{\prime}+\left(\tau_{2}+h_{2}\right) \widetilde{X}_{14}^{\prime} \\
& \widetilde{\Phi}_{15}=B L+d_{2} X_{15}^{\prime}+\tau_{2} \bar{X}_{15}^{\prime}+\left(\tau_{2}+h_{2}\right) \widetilde{X}_{15}^{\prime}, \\
& \widetilde{\Phi}_{16}=B_{h} L+d_{2} X_{16}^{\prime}+\tau_{2} \bar{X}_{16}^{\prime}+\left(\tau_{2}+h_{2}\right) \widetilde{X}_{16}^{\prime} \\
& \widetilde{\Phi}_{22}=-\bar{S}_{1}-\bar{N}_{2}^{T}-\bar{N}_{2}+d_{2} X_{22}^{\prime}+\tau_{2} \bar{X}_{22}^{\prime}+\left(\tau_{2}+h_{2}\right) \widetilde{X}_{22}^{\prime}, \\
& \widetilde{\Phi}_{23}=d_{2} X_{23}^{\prime}+\tau_{2} \bar{X}_{23}^{\prime}+\left(\tau_{2}+h_{2}\right) \widetilde{X}_{23}^{\prime}, \\
& \widetilde{\Phi}_{24}=d_{2} X_{24}^{\prime}+\tau_{2} \bar{X}_{24}^{\prime}+\left(\tau_{2}+h_{2}\right) \widetilde{X}_{24}^{\prime}, \\
& \widetilde{\Phi}_{25}=d_{2} X_{25}^{\prime}+\tau_{2} \bar{X}_{25}^{\prime}+\left(\tau_{2}+h_{2}\right) \widetilde{X}_{25}^{\prime} \text {, } \\
& \widetilde{\Phi}_{26}=d_{2} X_{26}^{\prime}+\tau_{2} \bar{X}_{26}^{\prime}+\left(\tau_{2}+h_{2}\right) \widetilde{X}_{26}^{\prime} \text {, } \\
& \widetilde{\Phi}_{33}=-\bar{S}_{2}-\bar{M}_{2}^{T}-\bar{M}_{2}+d_{2} X_{33}^{\prime}+\tau_{2} \bar{X}_{33}^{\prime}+\left(\tau_{2}+h_{2}\right) \widetilde{X}_{33}^{\prime}, \\
& \widetilde{\Phi}_{34}=h_{2} X_{34}^{\prime}+d_{2} \bar{X}_{34}^{\prime}+\left(\tau_{2}+h_{2}\right) \widetilde{X}_{34}^{\prime} \text {, } \\
& \widetilde{\Phi}_{35}=h_{2} X_{35}^{\prime}+d_{2} \bar{X}_{35}^{\prime}+\left(\tau_{2}+h_{2}\right) \widetilde{X}_{35}^{\prime} \text {, } \\
& \widetilde{\Phi}_{36}=h_{2} X_{36}^{\prime}+d_{2} \bar{X}_{36}^{\prime}+\left(\tau_{2}+h_{2}\right) \widetilde{X}_{36}^{\prime} \text {, } \\
& \widetilde{\Phi}_{44}=-\bar{S}_{3}-\bar{T}_{2}-\bar{T}_{2}^{T}+d_{2} X_{44}^{\prime}+\tau_{2} \bar{X}_{44}^{\prime}+\left(\tau_{2}+h_{2}\right) \widetilde{X}_{44}^{\prime}, \\
& \widetilde{\Phi}_{45}=d_{2} X_{45}^{\prime}+\tau_{2} \bar{X}_{45}^{\prime}+\left(\tau_{2}+h_{2}\right) \widetilde{X}_{45}^{\prime} \text {, } \\
& \widetilde{\Phi}_{46}=d_{2} X_{46}^{\prime}+\tau_{2} \bar{X}_{46}^{\prime}+\left(\tau_{2}+h_{2}\right) \widetilde{X}_{46}^{\prime} \text {, } \\
& \widetilde{\Phi}_{55}=\alpha_{1} I-2 \alpha_{1} L+d_{2} X_{55}^{\prime}+\tau_{2} \bar{X}_{55}^{\prime}+\left(\tau_{2}+h_{2}\right) \widetilde{X}_{55}^{\prime}, \\
& \widetilde{\Phi}_{56}=h_{2} X_{56}^{\prime}+\tau_{2} \bar{X}_{56}^{\prime}+\left(\tau_{2}+h_{2}\right) \widetilde{X}_{56}^{\prime} \text {, } \\
& \widetilde{\Phi}_{66}=\alpha_{2} I-2 \alpha_{2} L+d_{2} X_{66}^{\prime}+\tau_{2} \bar{X}_{66}^{\prime}+\left(\tau_{2}+h_{2}\right) \widetilde{X}_{66}^{\prime} \text {, } \\
& X^{\prime}=\left[\begin{array}{cccccc}
X_{11}^{\prime} & X_{12}^{\prime} & X_{13}^{\prime} & X_{14}^{\prime} & X_{15}^{\prime} & X_{16}^{\prime} \\
* & X_{22}^{\prime} & X_{23}^{\prime} & X_{24}^{\prime} & X_{25}^{\prime} & X_{26}^{\prime} \\
* & * & X_{33}^{\prime} & X_{34}^{\prime} & X_{35}^{\prime} & X_{36}^{\prime} \\
* & * & * & X_{44}^{\prime} & X_{45}^{\prime} & X_{46}^{\prime} \\
* & * & * & * & X_{55}^{\prime} & X_{56}^{\prime} \\
* & * & * & * & * & X_{66}^{\prime}
\end{array}\right],
\end{aligned}
$$




$$
\begin{aligned}
& \bar{X}^{\prime}=\left[\begin{array}{cccccc}
\bar{X}_{11}^{\prime} & \bar{X}_{12}^{\prime} & \bar{X}_{13}^{\prime} & \bar{X}_{14}^{\prime} & \bar{X}_{15}^{\prime} & \bar{X}_{16}^{\prime} \\
* & \bar{X}_{22}^{\prime} & \bar{X}_{23}^{\prime} & \bar{X}_{24}^{\prime} & \bar{X}_{25}^{\prime} & \bar{X}_{26}^{\prime} \\
* & * & \bar{X}_{33}^{\prime} & \bar{X}_{34}^{\prime} & \bar{X}_{35}^{\prime} & \bar{X}_{36}^{\prime} \\
* & * & * & \bar{X}_{44}^{\prime} & \bar{X}_{45}^{\prime} & \bar{X}_{46}^{\prime} \\
* & * & * & * & \bar{X}_{55}^{\prime} & \bar{X}_{56}^{\prime} \\
* & * & * & * & * & \bar{X}_{66}^{\prime}
\end{array}\right], \\
& \widetilde{X}^{\prime}=\left[\begin{array}{cccccc}
\widetilde{X}_{11}^{\prime} & \widetilde{X}_{12}^{\prime} & \widetilde{X}_{13}^{\prime} & \widetilde{X}_{14}^{\prime} & \widetilde{X}_{15}^{\prime} & \widetilde{X}_{16}^{\prime} \\
* & \widetilde{X}_{22}^{\prime} & \widetilde{X}_{23}^{\prime} & \widetilde{X}_{24}^{\prime} & \widetilde{X}_{25}^{\prime} & \widetilde{X}_{26}^{\prime} \\
* & * & \widetilde{X}_{33}^{\prime} & \widetilde{X}_{34}^{\prime} & \widetilde{X}_{35}^{\prime} & \widetilde{X}_{36}^{\prime} \\
* & * & * & \widetilde{X}_{44}^{\prime} & \widetilde{X}_{45}^{\prime} & \widetilde{X}_{46}^{\prime} \\
* & * & * & * & \widetilde{X}_{55}^{\prime} & \widetilde{X}_{56}^{\prime} \\
* & * & * & * & * & \widetilde{X}_{66}^{\prime}
\end{array}\right], \\
& \bar{N}=\left[\begin{array}{llllll}
\bar{N}_{1}^{T} & \bar{N}_{2}^{T} & 0 & 0 & 0 & 0
\end{array}\right]^{T}, \\
& \bar{M}=\left[\begin{array}{llllll}
\bar{M}_{1}^{T} & 0 & \bar{M}_{2}^{T} & 0 & 0 & 0
\end{array}\right]^{T}, \\
& \bar{T}=\left[\begin{array}{llllll}
\bar{T}_{1}^{T} & 0 & 0 & \bar{T}_{2}^{T} & 0 & 0
\end{array}\right]^{T} .
\end{aligned}
$$

Proof. From Theorem 5, if the closed-loop system is asymptotically stable, (14) holds. By Lemma 3, (14) is equivalent to

$$
\Phi=\left[\begin{array}{cccccccccc}
\Phi_{11} & \Phi_{12} & \Phi_{13} & \Phi_{14} & \Phi_{15} & \Phi_{16} & (A-I)^{T} P & d_{2}(A-I)^{T} Q_{1} & \tau_{2}(A-I)^{T} Q_{2} & \left(\tau_{2}+h_{2}\right)(A-I)^{T} Q_{3} \\
* & \Phi_{22} & \Phi_{23} & \Phi_{24} & \Phi_{25} & \Phi_{26} & A_{d}^{T} P & d_{2} A_{d}^{T} Q_{1} & \tau_{2} A_{d}^{T} Q_{2} & \left(\tau_{2}+h_{2}\right) A_{d}^{T} Q_{3} \\
* & * & \Phi_{33} & \Phi_{34} & \Phi_{35} & \Phi_{36} & (B K)^{T} P & d_{2}(B K)^{T} Q_{1} & \tau_{2}(B K)^{T} Q_{2} & \left(\tau_{2}+h_{2}\right)(B K)^{T} Q_{3} \\
* & * & * & \Phi_{44} & \Phi_{45} & \Phi_{46} & \left(B_{h} K\right)^{T} P & d_{2}\left(B_{h} K\right)^{T} Q_{1} & \tau_{2}\left(B_{h} K\right)^{T} Q_{2} & \left(\tau_{2}+h_{2}\right)\left(B_{h} K\right)^{T} Q_{3} \\
* & * & * & * & \Phi_{55} & \Phi_{56} & B^{T} P & d_{2} B^{T} Q_{1} & \tau_{2} B^{T} Q_{2} & \left(\tau_{2}+h_{2}\right) B^{T} Q_{3} \\
* & * & * & * & * & \Phi_{66} & B_{h}^{T} P & d_{2} B_{h}^{T} Q_{1} & \tau_{2} B_{h}^{T} Q_{2} & \left(\tau_{2}+h_{2}\right) B_{h}^{T} Q_{3} \\
* & * & * & * & * & * & -P & 0 & 0 & 0 \\
* & * & * & * & * & * & * & -d_{2} Q_{1} & 0 & 0 \\
* & * & * & * & * & * & * & * & -\tau_{2} Q_{2} & 0 \\
* & * & * & * & * & * & * & * & * & -\left(\tau_{2}+h_{2}\right) Q_{3}
\end{array}\right]
$$


where

$$
\begin{aligned}
\Phi_{11}= & +P(A-I)^{T}+(A-1)^{T} P+N_{1}^{T}+N_{1}+M_{1}^{T} \\
& +M_{1}+T_{1}^{T}+T_{1}+d_{2} X_{11}+\tau_{2} \bar{X}_{11} \\
& +\left(\tau_{2}+d_{2}\right) \widetilde{X}_{11} \\
\Phi_{12}= & P A_{d}-N_{1}+N_{2}^{T}+d_{2} X_{12}+\tau_{2} \bar{X}_{12} \\
& +\left(\tau_{2}+h_{2}\right) \widetilde{X}_{12} \\
\Phi_{13}= & P B K+N_{3}^{T}-M_{1}+M_{2}^{T}+d_{2} X_{13}+\tau_{2} \bar{X}_{13} \\
& +\left(\tau_{2}+h_{2}\right) \widetilde{X}_{13} \\
\Phi_{14}= & P B_{h} K-T_{1}+T_{2}^{T}+d_{2} X_{14}+\tau_{2} \bar{X}_{14} \\
& +\left(\tau_{2}+h_{2}\right) \widetilde{X}_{14}, \\
\Phi_{15}= & P B+d_{2} X_{15}+\tau_{2} \bar{X}_{15}+\left(\tau_{2}+h_{2}\right) \widetilde{X}_{15} \\
\Phi_{16}= & P B_{h}+d_{2} X_{16}+\tau_{2} \bar{X}_{16}+\left(\tau_{2}+h_{2}\right) \widetilde{X}_{16} \\
\Phi_{22}= & -S_{1}-N_{2}^{T}-N_{2}+d_{2} X_{22}+\tau_{2} \bar{X}_{22} \\
& +\left(\tau_{2}+h_{2}\right) \widetilde{X}_{22}, \\
\Phi_{23}= & d_{2} X_{23}+\tau_{2} \bar{X}_{23}+\left(\tau_{2}+h_{2}\right) \widetilde{X}_{23}, \\
\Phi_{24}= & d_{2} X_{24}+\tau_{2} \bar{X}_{24}+\left(\tau_{2}+h_{2}\right) \widetilde{X}_{24} \\
\Phi_{25}= & d_{2} X_{25}+\tau_{2} \bar{X}_{25}+\left(\tau_{2}+h_{2}\right) \widetilde{X}_{25} \\
\Phi_{26}= & d_{2} X_{26}+\tau_{2} \bar{X}_{26}+\left(\tau_{2}+h_{2}\right) \widetilde{X}_{26} \\
&
\end{aligned}
$$

$$
\begin{aligned}
\Phi_{33}= & -S_{2}-M_{2}^{T}-M_{2}+\alpha_{1} K^{T} K+d_{2} X_{33}+\tau_{2} \bar{X}_{33} \\
& +\left(\tau_{2}+h_{2}\right) \widetilde{X}_{33}, \\
\Phi_{34}= & d_{2} X_{34}+\tau_{2} \bar{X}_{34}+\left(\tau_{2}+h_{2}\right) \widetilde{X}_{34} \\
\Phi_{35}= & d_{2} X_{35}+\tau_{2} \bar{X}_{35}+\left(\tau_{2}+h_{2}\right) \widetilde{X}_{35} \\
\Phi_{36}= & d_{2} X_{36}+\tau_{2} \bar{X}_{36}+\left(\tau_{2}+h_{2}\right) \widetilde{X}_{36} \\
\Phi_{44}= & -S_{3}-T_{2}-T_{2}^{T}+\alpha_{2} K^{T} K+d_{2} X_{44}+\tau_{2} \bar{X}_{44} \\
& +\left(\tau_{2}+h_{2}\right) \widetilde{X}_{44}, \\
\Phi_{45}= & d_{2} X_{45}+\tau_{2} \bar{X}_{45}+\left(\tau_{2}+h_{2}\right) \widetilde{X}_{45} \\
\Phi_{46}= & d_{2} X_{46}+\tau_{2} \bar{X}_{46}+\left(\tau_{2}+h_{2}\right) \widetilde{X}_{46} \\
\Phi_{55}= & -\alpha_{1} I+d_{2} X_{55}+\tau_{2} \bar{X}_{55}+\left(\tau_{2}+h_{2}\right) \widetilde{X}_{55} \\
\Phi_{56}= & d_{2} X_{56}+\tau_{2} \bar{X}_{56}+\left(\tau_{2}+h_{2}\right) \widetilde{X}_{56}, \\
\Phi_{66}= & -\alpha_{2} I+d_{2} X_{66}+\tau_{2} \bar{X}_{66}+\left(\tau_{2}+h_{2}\right) \widetilde{X}_{66} .
\end{aligned}
$$

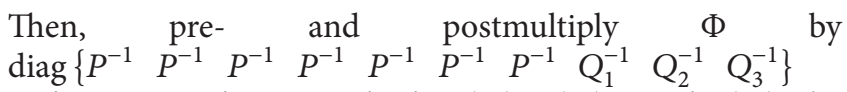
and pre- and postmultiply (17), (18), and (19) by $\operatorname{diag}\left\{\begin{array}{llllllll}P^{-1} & P^{-1} & P^{-1} & P^{-1} & P^{-1} & P^{-1} & P^{-1}\end{array}\right\} \quad$ similarly. Let $P^{-1}=L, K P^{-1}=R, \bar{S}_{i}=P^{-1} S_{i} P^{-1}, i=1,2,3, P^{-1} X P^{-1}=$ $X^{\prime}, P^{-1} \bar{X} P^{-1}=\bar{X}^{\prime}, P^{-1} \widetilde{X} P^{-1}=\widetilde{X}^{\prime}, P^{-1} N_{1,2} P^{-1}=$ $\bar{N}_{1,2}, P^{-1} M_{1,2} P^{-1}=\bar{M}_{1,2}, P^{-1} T_{1,2} P^{-1}=\bar{T}_{1,2}, Q_{i}=\varepsilon_{i} L^{-1}, i=$ $1,2,3$. Then, we have

$$
\begin{aligned}
& \bar{\Phi}=\left[\begin{array}{cccccccccc}
\bar{\Phi}_{11} & \bar{\Phi}_{12} & \bar{\Phi}_{13} & \bar{\Phi}_{14} & \bar{\Phi}_{15} & \bar{\Phi}_{16} & L(A-I)^{T} & d_{2} L(A-I)^{T} & \tau_{2} L(A-I)^{T} & \left(\tau_{2}+h_{2}\right) L(A-I)^{T} \\
* & \bar{\Phi}_{22} & \bar{\Phi}_{23} & \bar{\Phi}_{24} & \bar{\Phi}_{25} & \bar{\Phi}_{26} & L A_{d}^{T} & d_{2} L A_{d}^{T} & \tau_{2} L A_{d}^{T} & \left(\tau_{2}+h_{2}\right) L A_{d}^{T} \\
* & * & \bar{\Phi}_{33} & \bar{\Phi}_{34} & \bar{\Phi}_{35} & \bar{\Phi}_{36} & R^{T} B^{T} & d_{2} R^{T} B^{T} & \tau_{2} R^{T} B^{T} & \left(\tau_{2}+h_{2}\right) R^{T} B^{T} \\
* & * & * & \bar{\Phi}_{44} & \bar{\Phi}_{45} & \bar{\Phi}_{46} & R^{T} B_{d}^{T} & d_{2} R^{T} B_{d}^{T} & \tau_{2} R^{T} B_{d}^{T} & \left(\tau_{2}+h_{2}\right) R^{T} B_{d}^{T} \\
* & * & * & * & \bar{\Phi}_{55} & \bar{\Phi}_{56} & L B^{T} & d_{2} L B^{T} & \tau_{2} L B^{T} & \left(\tau_{2}+h_{2}\right) L B^{T} \\
* & * & * & * & * & \bar{\Phi}_{66} & L B_{d}^{T} & d_{2} L B_{d}^{T} & \tau_{2} L B_{d}^{T} & \left(\tau_{2}+h_{2}\right) L B_{d}^{T} \\
* & * & * & * & * & * & -L & 0 & 0 & 0 \\
* & * & * & * & * & * & * & -h_{2} \varepsilon_{1}^{-1} L & 0 & 0 \\
* & * & * & * & * & * & * & * & -\tau_{2} \varepsilon_{2}^{-1} L & 0 \\
* & * & * & * & * & * & * & * & * & -\tau_{2} \varepsilon_{2}^{-1} L
\end{array}\right]<0, \\
& \bar{\psi}_{1}=\left[\begin{array}{ccccccc}
X_{11}^{\prime} & X_{12}^{\prime} & X_{13}^{\prime} & X_{14}^{\prime} & X_{15}^{\prime} & X_{16}^{\prime} & \bar{N}_{1} \\
* & X_{22}^{\prime} & X_{23}^{\prime} & X_{24}^{\prime} & X_{25}^{\prime} & X_{26}^{\prime} & \bar{N}_{2} \\
* & * & X_{33}^{\prime} & X_{34}^{\prime} & X_{35}^{\prime} & X_{36}^{\prime} & 0 \\
* & * & * & X_{44}^{\prime} & X_{45}^{\prime} & X_{46}^{\prime} & 0 \\
* & * & * & * & X_{55}^{\prime} & X_{56}^{\prime} & 0 \\
* & * & * & * & * & X_{66}^{\prime} & 0 \\
* & * & * & * & * & * & \varepsilon_{1} L
\end{array}\right]>0,
\end{aligned}
$$




$$
\begin{aligned}
& \bar{\psi}_{2}=\left[\begin{array}{ccccccc}
\bar{X}_{11}^{\prime} & \bar{X}_{12}^{\prime} & \bar{X}_{13}^{\prime} & \bar{X}_{14}^{\prime} & \bar{X}_{15}^{\prime} & \bar{X}_{16}^{\prime} & \bar{M}_{1} \\
* & \bar{X}_{22}^{\prime} & \bar{X}_{23}^{\prime} & \bar{X}_{24}^{\prime} & \bar{X}_{25}^{\prime} & \bar{X}_{26}^{\prime} & 0 \\
* & * & \bar{X}_{33}^{\prime} & \bar{X}_{34}^{\prime} & \bar{X}_{35}^{\prime} & \bar{X}_{36}^{\prime} & \bar{M}_{2} \\
* & * & * & \bar{X}_{44}^{\prime} & \bar{X}_{45}^{\prime} & \bar{X}_{46}^{\prime} & 0 \\
* & * & * & * & \bar{X}_{55}^{\prime} & \bar{X}_{56}^{\prime} & 0 \\
* & * & * & * & * & \bar{X}_{66}^{\prime} & 0 \\
* & * & * & * & * & * & \varepsilon_{2} L
\end{array}\right]>0, \\
& \widetilde{\psi}_{3}=\left[\begin{array}{ccccccc}
\widetilde{X}_{11}^{\prime} & \widetilde{X}_{12}^{\prime} & \widetilde{X}_{13}^{\prime} & \widetilde{X}_{14}^{\prime} & \widetilde{X}_{15}^{\prime} & \widetilde{X}_{16}^{\prime} & \bar{T}_{1} \\
* & \widetilde{X}_{22}^{\prime} & \widetilde{X}_{23}^{\prime} & \widetilde{X}_{24}^{\prime} & \widetilde{X}_{25}^{\prime} & \widetilde{X}_{26}^{\prime} & 0 \\
* & * & \widetilde{X}_{33}^{\prime} & \widetilde{X}_{34}^{\prime} & \widetilde{X}_{35}^{\prime} & \widetilde{X}_{36}^{\prime} & 0 \\
* & * & * & \widetilde{X}_{44}^{\prime} & \widetilde{X}_{45}^{\prime} & \widetilde{X}_{46}^{\prime} & \bar{T}_{2} \\
* & * & * & * & \widetilde{X}_{55}^{\prime} & \widetilde{X}_{56}^{\prime} & 0 \\
* & * & * & * & * & \widetilde{X}_{66}^{\prime} & 0 \\
* & * & * & * & * & * & \varepsilon_{3} L
\end{array}\right]>0,
\end{aligned}
$$

where

$$
\begin{aligned}
\bar{\Phi}_{11}= & \left(d_{2}-d_{1}+1\right) \bar{S}_{1}+\left(\tau_{2}-\tau_{1}+1\right) \bar{S}_{2} \\
& +\left(\left(\tau_{2}+h_{2}\right)-\left(\tau_{1}+h_{1}\right)+1\right) \bar{S}_{3}+(A-I) L \\
& +L(A-1)^{T}+\bar{N}_{1}^{T}+\bar{N}_{1}+\bar{M}_{1}^{T}+\bar{M}_{1}+\bar{T}_{1}^{T} \\
& +\bar{T}_{1}+d_{2} X_{11}^{\prime}+\tau_{2} \bar{X}_{11}^{\prime}+\left(\tau_{2}+h_{2}\right) \widetilde{X}_{11}^{\prime}, \\
\bar{\Phi}_{12}= & A_{h} L-\bar{N}_{1}+\bar{N}_{2}^{T}+d_{2} X_{12}^{\prime}+\tau_{2} \bar{X}_{12}^{\prime} \\
& +\left(\tau_{2}+h_{2}\right) \widetilde{X}_{12}^{\prime}, \\
\bar{\Phi}_{13}= & B R-\bar{M}_{1}+\bar{M}_{2}^{T}+d_{2} X_{13}^{\prime}+\tau_{2} \bar{X}_{13}^{\prime} \\
& +\left(\tau_{2}+h_{2}\right) \widetilde{X}_{13}^{\prime}, \\
\bar{\Phi}_{14}= & B_{d} R-\bar{T}_{1}+\bar{T}_{2}^{T}+d_{2} X_{14}^{\prime}+\tau_{2} \bar{X}_{14}^{\prime} \\
& +\left(\tau_{2}+h_{2}\right) \widetilde{X}_{14}^{\prime}, \\
\bar{\Phi}_{15}= & B L+d_{2} X_{15}^{\prime}+\tau_{2} \bar{X}_{15}^{\prime}+\left(\tau_{2}+h_{2}\right) \widetilde{X}_{15}^{\prime}, \\
\bar{\Phi}_{16}= & B_{d} L+d_{2} X_{16}^{\prime}+\tau_{2} \bar{X}_{16}^{\prime}+\left(\tau_{2}+h_{2}\right) \widetilde{X}_{16}^{\prime}, \\
\bar{\Phi}_{22}= & -\bar{S}_{1}-\bar{N}_{2}^{T}-\bar{N}_{2}+d_{2} X_{22}^{\prime}+\tau_{2} \bar{X}_{22}^{\prime} \\
& +\left(\tau_{2}+h_{2}\right) \widetilde{X}_{22}^{\prime}, \\
\bar{\Phi}_{23}= & d_{2} X_{23}^{\prime}+\tau_{2} \bar{X}_{23}^{\prime}+\left(\tau_{2}+h_{2}\right) \widetilde{X}_{23}^{\prime},
\end{aligned}
$$

$\bar{\Phi}_{24}=d_{2} X_{24}^{\prime}+\tau_{2} \bar{X}_{24}^{\prime}+\left(\tau_{2}+h_{2}\right) \widetilde{X}_{24}^{\prime}$,

$\bar{\Phi}_{25}=d_{2} X_{25}^{\prime}+\tau_{2} \bar{X}_{25}^{\prime}+\left(\tau_{2}+h_{2}\right) \widetilde{X}_{25}^{\prime}$,

$\bar{\Phi}_{26}=d_{2} X_{26}^{\prime}+\tau_{2} \bar{X}_{26}^{\prime}+\left(\tau_{2}+h_{2}\right) \widetilde{X}_{26}^{\prime}$,

$\bar{\Phi}_{33}=-\bar{S}_{2}-\bar{M}_{2}^{T}-\bar{M}_{2}+\alpha_{1} L^{T} L+h_{2} X_{33}^{\prime}+\tau_{2} \bar{X}_{33}^{\prime}$

$+\left(\tau_{2}+d_{2}\right) \widetilde{X}_{33}^{\prime}$,

$\bar{\Phi}_{34}=d_{2} X_{34}^{\prime}+\tau_{2} \bar{X}_{34}^{\prime}+\left(\tau_{2}+h_{2}\right) \widetilde{X}_{34}^{\prime}$,

$\bar{\Phi}_{35}=d_{2} X_{35}^{\prime}+\tau_{2} \bar{X}_{35}^{\prime}+\left(\tau_{2}+h_{2}\right) \widetilde{X}_{35}^{\prime}$,

$\bar{\Phi}_{36}=d_{2} X_{36}^{\prime}+\tau_{2} \bar{X}_{36}^{\prime}+\left(\tau_{2}+h_{2}\right) \widetilde{X}_{36}^{\prime}$,

$\bar{\Phi}_{44}=-\bar{S}_{3}-\bar{T}_{2}-\bar{T}_{2}^{T}+\alpha_{2} L^{T} L+d_{2} X_{44}^{\prime}+\tau_{2} \bar{X}_{44}^{\prime}$

$$
+\left(\tau_{2}+h_{2}\right) \widetilde{X}_{44}^{\prime}
$$

$\bar{\Phi}_{45}=d_{2} X_{45}^{\prime}+\tau_{2} \bar{X}_{45}^{\prime}+\left(\tau_{2}+h_{2}\right) \widetilde{X}_{45}^{\prime}$,

$\bar{\Phi}_{46}=d_{2} X_{46}^{\prime}+\tau_{2} \bar{X}_{46}^{\prime}+\left(\tau_{2}+h_{2}\right) \widetilde{X}_{46}^{\prime}$,

$\bar{\Phi}_{55}=-\alpha_{1} L^{T} L+d_{2} X_{55}^{\prime}+\tau_{2} \bar{X}_{55}^{\prime}+\left(\tau_{2}+h_{2}\right) \widetilde{X}_{55}^{\prime}$,

$\bar{\Phi}_{56}=h_{2} X_{56}^{\prime}+\tau_{2} \bar{X}_{56}^{\prime}+\left(\tau_{2}+d_{2}\right) \widetilde{X}_{56}^{\prime}$,

$\bar{\Phi}_{66}=-\alpha_{2} L^{T} L+d_{2} X_{66}^{\prime}+\tau_{2} \bar{X}_{66}^{\prime}+\left(\tau_{2}+h_{2}\right) \widetilde{X}_{66}^{\prime}$, 
and $X_{i j}, \bar{X}_{i j}, \widetilde{X}_{i j}, i, j=1, \ldots, 6$, are matrices with appropriate dimensions. During the proof, there exist nonlinear items $-\alpha_{i} L^{T} L, i=1,2$. Obviously, we can have $(I-L)^{T}(I-L) \geq 0$; then we obtain $I-2 L+L^{T} L \geq 0$. Thus, the nonlinear items satisfy the following formula:

$$
\begin{aligned}
& -\alpha_{1} L^{T} L \leq \alpha_{1} I-2 \alpha_{1} L, \\
& -\alpha_{2} L^{T} L \leq \alpha_{2} I-2 \alpha_{2} L .
\end{aligned}
$$

Then by Lemma 3, (33) can be obtained. Above all, if LMIs (33) and (34) hold, we can have $\Delta V(k)<0$. The proof is completed.

\section{Numerical Simulation}

In this section, in order to demonstrate the validity of the proposed method, numerical examples are given to illustrate the effectiveness of the developed results.

Example 1. Consider the following system as

$$
\begin{aligned}
A & =\left[\begin{array}{cc}
1 & 0 \\
0.25 & 0.4
\end{array}\right], \\
A_{d} & =\left[\begin{array}{ll}
-0.2 & 0.1 \\
0 & 0.1
\end{array}\right], \\
B & =\left[\begin{array}{ll}
0.3 & 0.1 \\
0.2 & 0.3
\end{array}\right], \\
B_{h} & =\left[\begin{array}{ll}
0.2 & 0.1 \\
0.1 & 0.4
\end{array}\right], \\
d_{2} & =4, \\
d_{1} & =1, \\
\tau_{2} & =4 \\
\tau_{1} & =1 \\
h_{2} & =3 \\
h_{1} & =1 .
\end{aligned}
$$

Choosing $\alpha_{1}=0.5, \alpha_{2}=0.5, \varepsilon_{1}=2, \varepsilon_{2}=2, \varepsilon_{3}=2, u_{\max }=$ 0.1 and using Matlab LMI toolbox to solve LMIs (33) and (34), we can obtain that the LMIs are feasible with

$$
\begin{aligned}
& R=\left[\begin{array}{cc}
-0.0033 & -0.0006 \\
-0.0006 & 0.0029
\end{array}\right], \\
& P=\left[\begin{array}{cc}
17.8771 & 4.3888 \\
4.3888 & 18.7636
\end{array}\right], \\
& K=\left[\begin{array}{cc}
-0.0610 & -0.0249 \\
0.0029 & 0.0527
\end{array}\right] .
\end{aligned}
$$
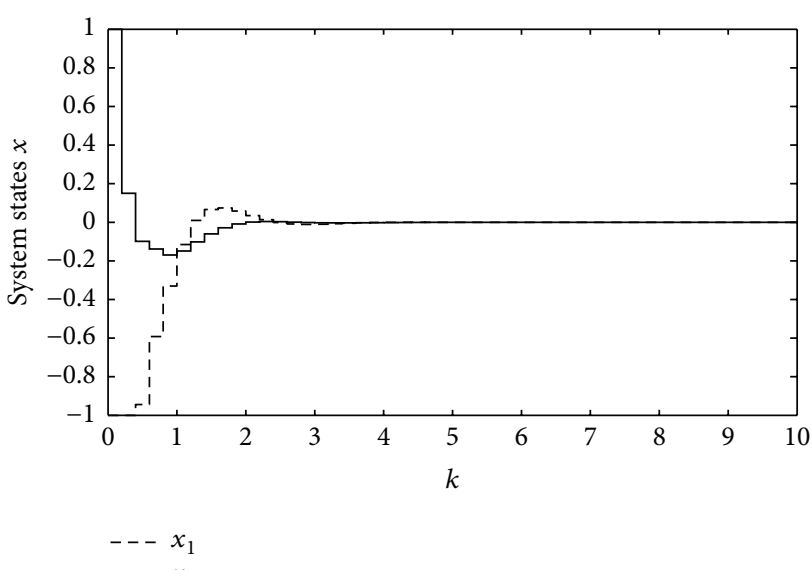

FIGURE 2: State trajectory of $x$.

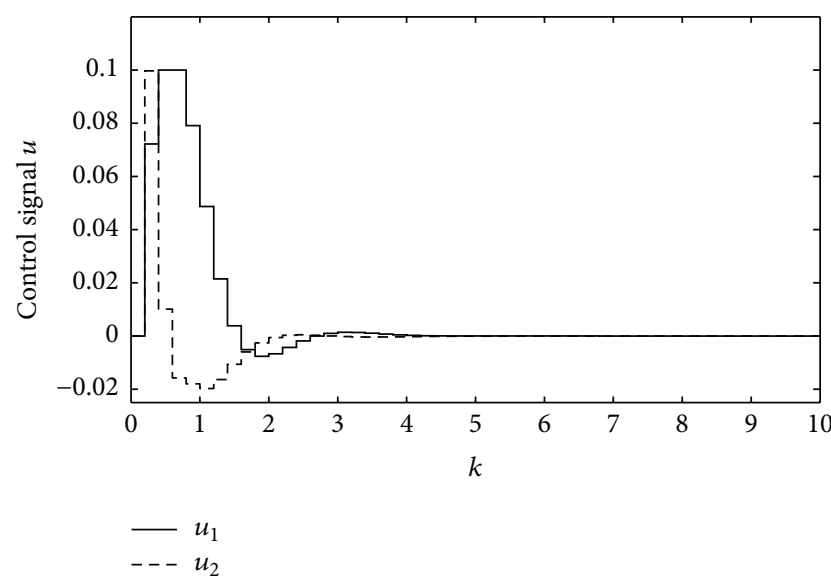

FIGURE 3: Control signal $u$ obtained by Theorem 6 .

According to Theorem 6, a state feedback controller can be constructed as

$$
\begin{aligned}
u(k) & =2 K x(k-\tau(k)) \\
& =\left[\begin{array}{cc}
-0.1220 & -0.0498 \\
0.0057 & 0.1054
\end{array}\right] x(k-\tau(k)) .
\end{aligned}
$$

With an initial condition $x(0)=[1 ;-1]$, simulation results are shown as follows. The state trajectories of the closed-loop system are shown in Figure 2 and Figure 3 shows the control signal response.

It can be clearly seen that the close-loop system is stabilized under the controller obtained by Theorem 6 and the control signal satisfies the actuator saturation.

Example 2. Consider the following system as

$$
\begin{aligned}
A & =\left[\begin{array}{ccc}
0 & 0.1 & 0.1 \\
0.1 & -0.2 & 0.1 \\
0.1 & 0.1 & 0.1
\end{array}\right], \\
A_{h} & =\left[\begin{array}{ccc}
0 & 0.1 & 0 \\
-0.1 & -0.1 & 0.1 \\
0.1 & 0.2 & 0.1
\end{array}\right],
\end{aligned}
$$




$$
\begin{aligned}
B & =\left[\begin{array}{ccc}
0.1 & 0.1 & 0 \\
0.2 & 0.3 & -0.1 \\
0 & 0.2 & -0.1
\end{array}\right], \\
B_{d} & =\left[\begin{array}{ccc}
0.2 & 0.1 & -0.1 \\
0.1 & 0.4 & 0 \\
-0.1 & 0.3 & -0.1
\end{array}\right], \\
d_{2} & =4 \\
d_{1} & =1 \\
\tau_{2} & =4 \\
\tau_{1} & =1 \\
h_{2} & =3 \\
h_{1} & =1 .
\end{aligned}
$$

Choosing $\alpha_{1}=0.5, \alpha_{2}=0.5, \varepsilon_{1}=2, \varepsilon_{2}=2, \varepsilon_{3}=2, u_{\max }=$ 0.1 and by using Matlab LMI toolbox, we have the following result:

$$
\begin{aligned}
& R=\left[\begin{array}{ccc}
-0.0015 & -0.0023 & -0.0143 \\
-0.00023 & 0.0040 & 0.0030 \\
-0.0143 & 0.0030 & -0.0279
\end{array}\right], \\
& P=\left[\begin{array}{ccc}
23.5668 & 9.2097 & -3.7498 \\
9.2097 & 38.4496 & -6.0510 \\
-3.7498 & -6.0510 & 9.3252
\end{array}\right], \\
& K=\left[\begin{array}{ccc}
-0.0016 & -0.0145 & -0.1145 \\
-0.0283 & 0.1142 & 0.0121 \\
-0.2064 & 0.1501 & -0.2283
\end{array}\right] .
\end{aligned}
$$

According to Theorem 6, a state feedback controller can be constructed as

$$
\begin{aligned}
u(k) & =2 K x(k-\tau(k)) \\
& =\left[\begin{array}{ccc}
0.0032 & -0.0290 & -0.2290 \\
-0.0566 & 0.02284 & 0.0241 \\
-0.4129 & 0.3003 & -0.4477
\end{array}\right] x(k-\tau(k)) .
\end{aligned}
$$

With an initial condition $x(0)=[-1 ; 1 ; 0]$, the corresponding results are displayed in Figures 4 and 5 which indicate that the closed-loop system can be stabilized and the saturation constraint is satisfied.

From the above simulation results, we can conclude that the developed state feedback stabilization is valid for NCSs subject to actuator saturation and induced delays.

\section{Conclusion}

In this paper, the stabilization problem has been developed for the NCSs with network-induced delays and actuator

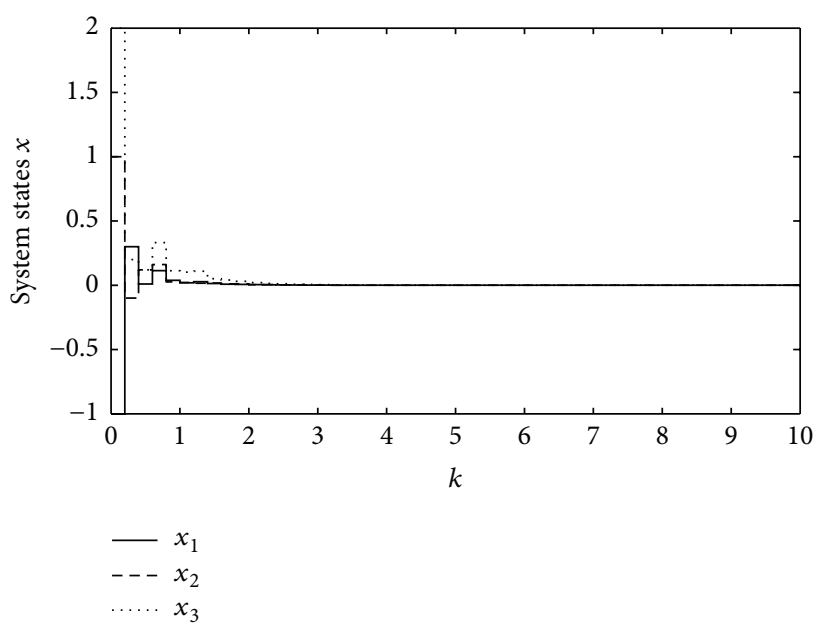

FIGURE 4: State trajectory of $x$.

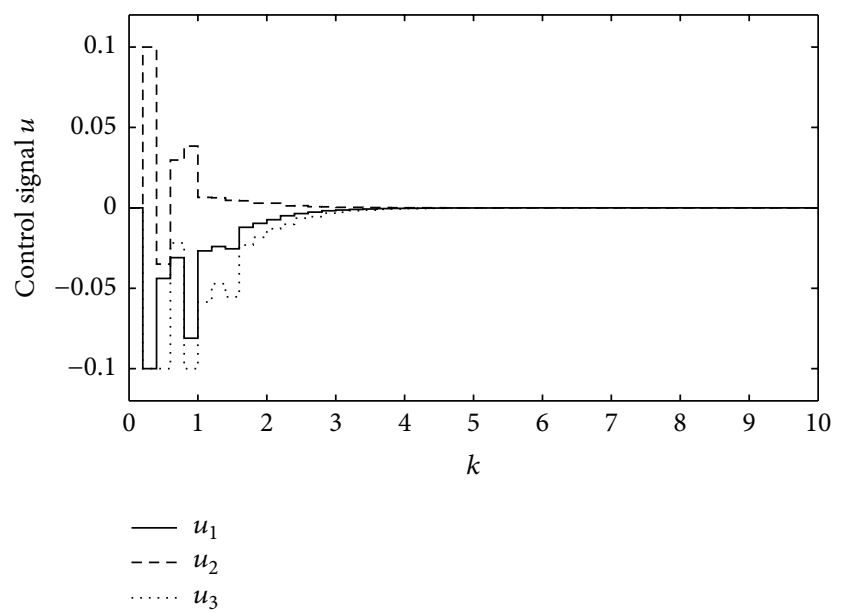

FIGURE 5: Control signal $u$ obtained by Theorem 6 .

saturation. State delay and input delay of control plant have been both considered. Lyapunov-Krasovskii theory and free weighting matrix method are used during the proof. Sufficient conditions in terms of LMIs are derived to ensure the existence of a state feedback controller which can stabilize the system. Numerical simulations are given to illustrate the necessity and the effectiveness of the proposed approach.

\section{Competing Interests}

The authors declare that they have no competing interests.

\section{Acknowledgments}

This work is partially supported by Jiangsu Natural Science Foundation of China (Grant no. SBK20130033), Six Talents Peak Project of Jiangsu Province (Grant no. 2012-XXRJ010), and Fundamental Research Funds for the Central Universities (Grant no. NE2016101). 


\section{References}

[1] Y. Halevi and A. Ray, "Integrated communication and control systems: part 1—analysis," Journal of Dynamic Systems, Measurement and Control, vol. 110, no. 4, pp. 367-373, 1988.

[2] J. Nillson, Real-time control systems with delays [Ph.D. thesis], Automatic Control Department, Lund Institute of Technology, Lund, Sweden, 1998.

[3] Y. Tipsuwan and M.-Y. Chow, "Control methodologies in networked control systems," Control Engineering Practice, vol. 11, no. 10, pp. 1099-1111, 2003.

[4] H. Gao, T. Chen, and J. Lam, "A new delay system approach to network-based control," Automatica, vol. 44, no. 1, pp. 39-52, 2008.

[5] K. Liu and E. Fridman, "Discrete-time network-based control under scheduling and actuator constraints," International Journal of Robust and Nonlinear Control, vol. 25, no. 12, pp. 18161830, 2015.

[6] M. Huzmezan, W. A. Gough, G. A. Dumont, and S. Kovac, "Time delay integrating systems: a challenge for process control industries. A practical solution," Control Engineering Practice, vol. 10, no. 10, pp. 1153-1161, 2002.

[7] H. Wu, K. S. Tsakalis, and G. T. Heydt, "Evaluation of time delay effects to wide area power system stabilizer design," IEEE Transactions on Power Systems, vol. 19, no. 4, pp. 1935-1941, 2004.

[8] G. Guo and L. Wang, "Control over medium-constrained vehicular networks with fading channels and random access protocol: a networked systems approach," IEEE Transactions on Vehicular Technology, vol. 64, no. 8, pp. 3347-3358, 2015.

[9] J.-P. Richard, "Time-delay systems: an overview of some recent advances and open problems," Automatica, vol. 39, no. 10, pp. 1667-1694, 2003.

[10] B.-F. Wang and G. Guo, "State estimation for discrete-time systems with Markovian time-delay and packet loss," Control Theory and Applications, vol. 26, no. 12, pp. 1331-1336, 2009.

[11] H. Wang, X. Wang, X.-J. Zhu, and X.-H. Wang, "Linear feedback controller design method for time-delay chaotic systems," Nonlinear Dynamics, vol. 70, no. 1, pp. 355-362, 2012.

[12] G. Guo, "Feedback control with scheduled communication sequences and random delays," International Journal of Control, Automation \& Systems, vol. 8, no. 6, pp. 1342-1348, 2010.

[13] S. Hu and Q. Zhu, "Stochastic optimal control and analysis of stability of networked control systems with long delay," Automatica, vol. 39, no. 11, pp. 1877-1884, 2003.

[14] L. Zhang, Y. Shi, T. Chen, and B. Huang, "A new method for stabilization of networked control systems with random delays," IEEE Transactions on Automatic Control, vol. 50, no. 8, pp. 11771181, 2005.

[15] F. Yang, Z. Wang, D. W. Ho, and M. Gani, "Robust $H_{\infty}$ control with missing measurements and time delays," IEEE Transactions on Automatic Control, vol. 52, no. 9, pp. 1666-1672, 2007.

[16] C. Lin, Z. D. Wang, and F. W. Yang, "Observer-based networked control for continuous-time systems with random sensor delays," Automatica, vol. 45, no. 2, pp. 578-584, 2009.

[17] G. Guo and H. Jin, "A switching system approach to actuator assignment with limited channels," International Journal of Robust \& Nonlinear Control, vol. 20, no. 12, pp. 1407-1426, 2010.

[18] B.-J. Yang, A. J. Calise, and J. I. Craig, "Adaptive output feedback control with input saturation," in Proceedings of the American Control Conference, pp. 1572-1577, June 2003.

[19] B. Zhou, Z. L. Lin, and G. R. Duan, "Stabilization of linear systems with input delay and saturation-a parametric Lyapunov equation approach," International Journal of Robust \& Nonlinear Control, vol. 20, no. 13, pp. 1502-1519, 2010.

[20] T. Hu, A. R. Teel, and L. Zaccarian, "Stability and performance for saturated systems via quadratic and nonquadratic Lyapunov functions," IEEE Transactions on Automatic Control, vol. 51, no. 11, pp. 1770-1786, 2006.

[21] T. Hu, A. R. Teel, and L. Zaccarian, "Anti-windup synthesis for linear control systems with input saturation: achieving regional, nonlinear performance," Automatica, vol. 44, no. 2, pp. 512-519, 2008.

[22] M. Chen, Q. Wu, C. Jiang, and B. Jiang, "Guaranteed transient performance based control with input saturation for near space vehicles," Science China. Information Sciences, vol. 57, no. 5, pp. 1-12, 2014.

[23] H. Fang, Z. Lin, and T. Hu, "Analysis of linear systems in the presence of actuator saturation and $L_{2}$-disturbances," Automatica, vol. 40, no. 7, pp. 1229-1238, 2004.

[24] Q. Y. Yang and M. Chen, "Robust control for uncertain linear system subject to input saturation," Journal of Applied Mathematics, vol. 2014, Article ID 803842, 12 pages, 2014.

[25] Q. Y. Yang and M. Chen, "Roubust adaptive tracking control for uncertain nonlinear systems with input saturation and unkown external disturbances," International Journal of Innovative Computing, Information and Control-Express Letters, vol. 9, no. 11, pp. 2973-2979, 2015.

[26] M. Chen, B. Jiang, and R. Cui, "Actuator fault-tolerant control of ocean surface vessels with input saturation," International Journal of Robust \& Nonlinear Control, vol. 26, no. 3, pp. 542564, 2016.

[27] Z. Zuo, D. W. C. Ho, and Y. Wang, "Fault tolerant control for singular systems with actuator saturation and nonlinear perturbation," Automatica, vol. 46, no. 3, pp. 569-576, 2010.

[28] Y. Wang, C. Wang, and Z. Zuo, "Controller synthesis for Markovian jump systems with incomplete knowledge of transition probabilities and actuator saturation," Journal of the Franklin Institute, vol. 348, no. 9, pp. 2417-2429, 2011.

[29] L. Lu, Z. Lin, and A. Bateman, "Decentralised state feedback design for large-scale linear systems subject to input saturation," IET Control Theory \& Applications, vol. 4, no. 2, pp. 206-227, 2010.

[30] A. Wei and Y. Wang, "Stabilization and $H_{\infty}$ control of nonlinear port-controlled Hamiltonian systems subject to actuator saturation," Automatica, vol. 46, no. 12, pp. 2008-2013, 2010.

[31] G. Guo and W. Yue, "Adaptive control with guaranteed contraction rate for systems with actuator saturation," Circuits, Systems, and Signal Processing, vol. 31, no. 4, pp. 1559-1576, 2012.

[32] X. Wang, Z. Liu, and Y. Cai, "Adaptive single neural network control for a class of uncertain discrete-time nonlinear strictfeedback systems with input saturation," Nonlinear Dynamics, vol. 82, no. 4, pp. 2021-2030, 2015.

[33] Y.-G. Sun and S.-Y. Qin, "Stability and stabilization of networked control systems with bounded packet dropout," Acta Automatica Sinica, vol. 37, no. 1, pp. 113-117, 2011.

[34] S. Wen, Z. Zeng, and T. Huang, "Observer-based $H_{\infty}$ control of discrete time-delay systems with random communication packet losses and multiplicative noises," Applied Mathematics and Computation, vol. 219, no. 12, pp. 6484-6493, 2013.

[35] X. J. Wei, H. F. Zhang, and L. Guo, "Saturating composite disturbance-observer-based control and $H_{\infty}$ control for discrete time-delay systems with nonlinearity," International Journal of Control, Automation, and Systems, vol. 7, no. 5, pp. 691-701, 2009. 


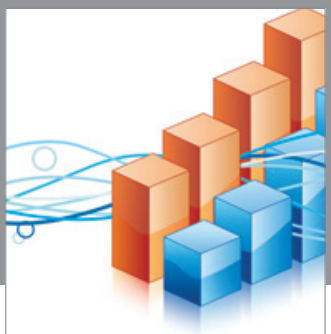

Advances in

Operations Research

vatem alat4

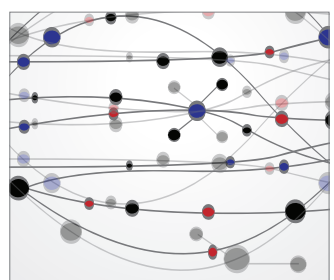

\section{The Scientific} World Journal
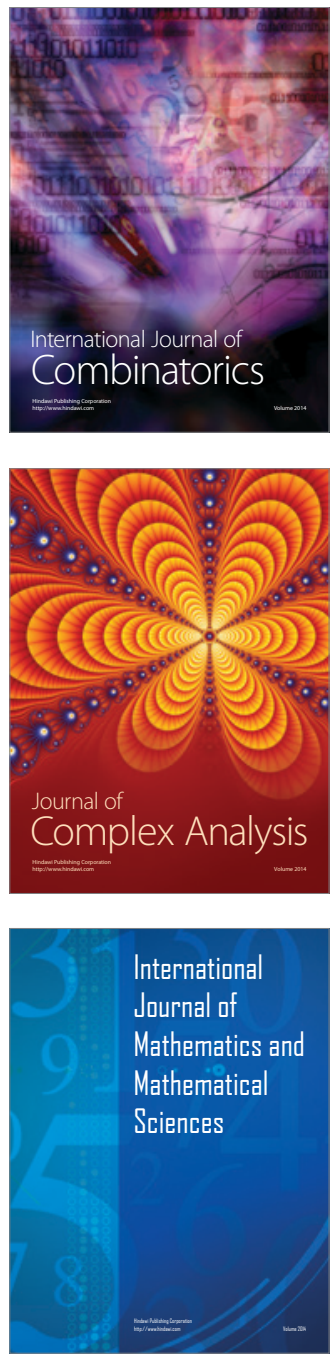
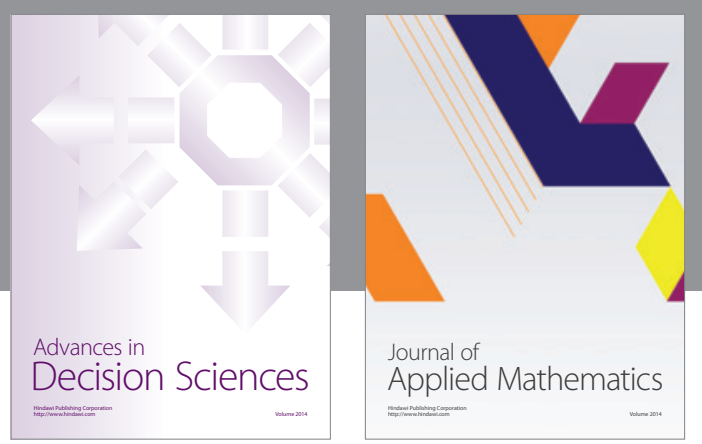

Algebra

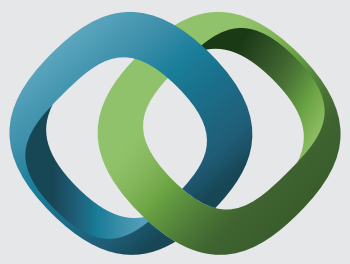

\section{Hindawi}

Submit your manuscripts at

http://www.hindawi.com
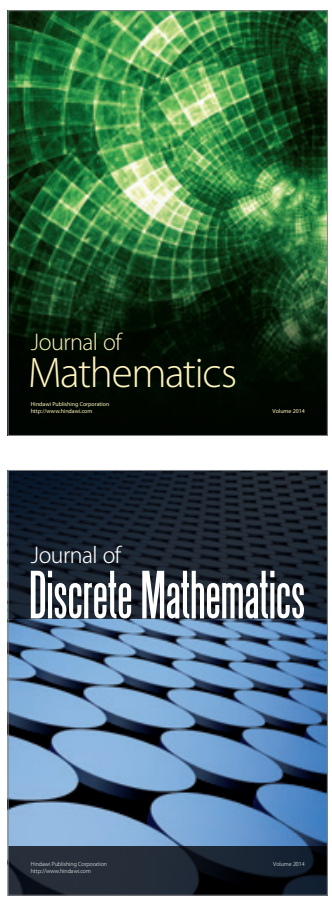

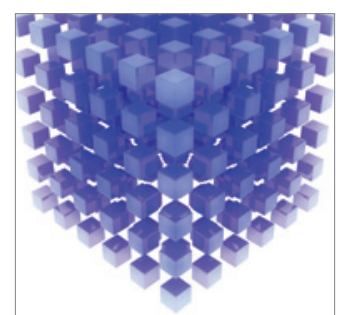

Mathematical Problems in Engineering
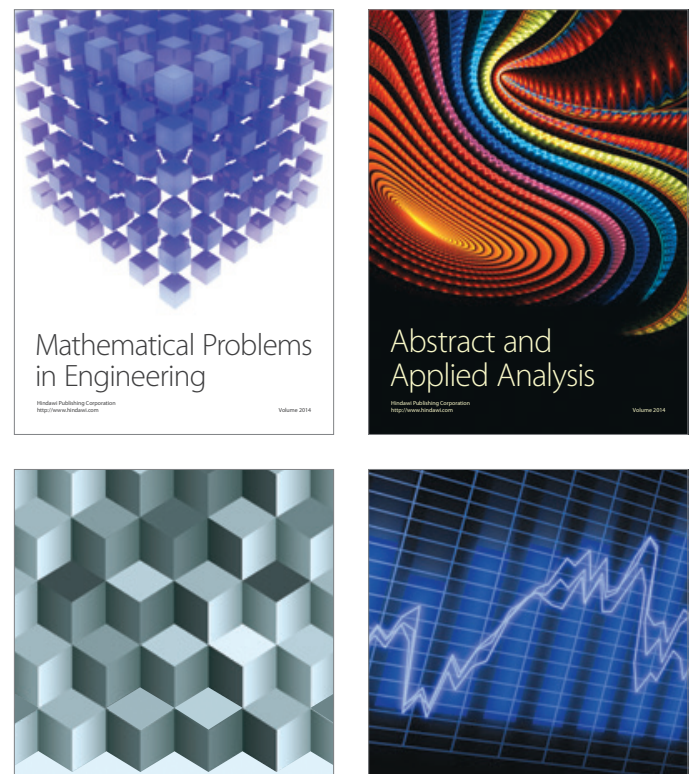

Journal of

Function Spaces

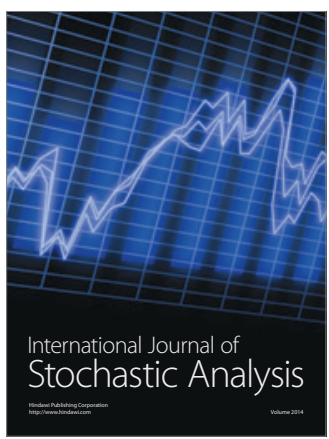

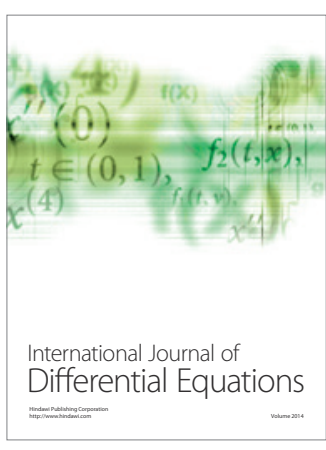
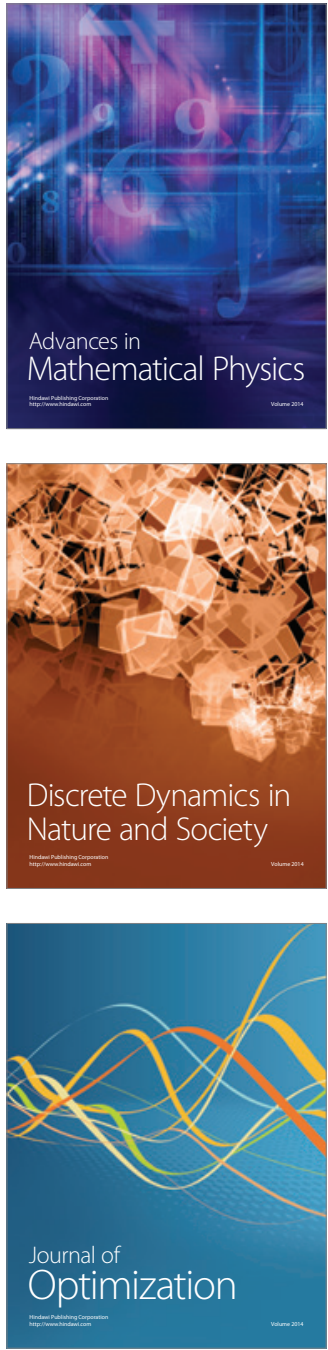